\title{
Vertex operator algebras and the zeta function
}

\author{
J. Lepowsky \\ Dedicated to Howard Garland on the occasion of his sixtieth \\ birthday
}

\begin{abstract}
We announce a new type of "Jacobi identity" for vertex operator algebras, incorporating values of the Riemann zeta function at negative integers. Using this we "explain" and generalize some recent work of S. Bloch's relating values of the zeta function with the commutators of certain operators and Lie algebras of differential operators.
\end{abstract}

\section{Introduction}

Consider the famous classical "formula"

$$
1+2+3+\cdots=-\frac{1}{12}
$$

which has the rigorous meaning

$$
\zeta(-1)=-\frac{1}{12}
$$

Here $\zeta$ is of course the Riemann zeta function

$$
\zeta(s)=\sum_{n>0} n^{-s}
$$

(analytically continued), and (11.1) is classically generalized by the formal equality

$$
\sum_{n>0} n^{s}=\zeta(-s)
$$


for $s=0,1,2, \ldots$ The classical number theory behind this analytic continuation is well known to be related to the widely-pervasive issue of regularizing certain infinities in quantum field theory, in particular, in conformal field theory. Here we shall announce some general principles of vertex operator algebra theory that elucidate the passage from the unrigorous but suggestive formula (1.1) to formula (1.2), and the generalization (1.4). In the process, we shall "explain" some recent work of S. Bloch's involving zeta-values and differential operators. The work [L2] contains details and related results. The material that we shall present involves foundational notions of vertex operator algebra theory, and we shall try to make this writeup accessible to nonspecialists by reviewing elementary matters.

We were motivated by a desire to understand some very interesting phenomena found by Bloch [B] relating the values $\zeta(-n), n=1,3,5, \ldots$, of the zeta function at negative odd integers to the commuatators of certain operators on an infinite-dimensional space. We shall begin with some elementary background and a brief description of this work, then we shall explain how to recover and somewhat generalize these results using vertex operator algebra theory, and finally, we shall place these ideas and results into a very general context and present some new general results in vertex operator algebra theory. These methods serve incidentally to enhance the many already-existing motivations for vertex operator algebra theory (see [Bo], [FLM]) and its underlying formal calculus (as developed in [FLM] and [FHL]).

One of our main themes is to "always" use generating functions - to introduce new formal variables and generating functions in order to try to make complicated things easier and more natural and at the same time, much more general, as in the corresponding parts of [FLM. We use commuting formal variables rather than complex variables because they provide the most natural way to handle the doubly-infinite series such as $\delta(x)=\sum_{n \in \mathbb{Z}} x^{n}$ that pervade the natural formulations and proofs. Other central themes are to exploit the formal exponential of the differential operator $x \frac{d}{d x}$ as a formal change-of-variables automorphism (again as in [FLM]); to formulate Euler's interpretation of the divergent series (1.4) by means of the operator product expansion in conformal field theory; and to place considerations about Lie algebras of differential operators into the very general context of what we termed the "Jacobi identity" [FLM] for vertex operator algebras. There are some interesting points of contact between the present work and [KR], [M] and [FKRW]. 
I am very pleased to dedicate this paper to Howard Garland on the occasion of his sixtieth birthday. This work was presented in a talk at Yale University in fall, 1997 at a seminar in his honor. I would like to mention here that it was from Howard Garland that Robert Wilson and I, in 1980, first learned about the idea of using formal delta-function calculus, which was also used in DKM]; cf. [FLM]. This was one among many of Howard's insights that have influenced us.

This work was also presented in a talk at the May, 1998 Conference on Representations of Affine and Quantum Affine Algebras and Related Topics at North Carolina State University. I would like to thank Naihuan Jing and Kailash Misra, the organizers, for a stimulating conference.

I am very grateful to Spencer Bloch for informing me about his work and for many valuable discussions.

This work was partially supported by NSF grants DMS-9401851 and DMS-9701150.

\section{Background}

Consider the commutative associative algebra $\mathbb{C}\left[t, t^{-1}\right]$ of Laurent polynomials in an indeterminate $t$, and consider its Lie algebra $\mathfrak{d}$ of derivations:

$$
\mathfrak{d}=\operatorname{Der} \mathbb{C}\left[t, t^{-1}\right],
$$

the Lie algebra of formal vector fields on the circle, with basis $\left\{t^{n} D \mid n \in \mathbb{Z}\right\}$, where

$$
D=D_{t}=t \frac{d}{d t}
$$

(A preview of one of our main themes: The "homogeneous" differential operator $D_{t}$, rather than $\frac{d}{d t}$, will be the appropriate form of differentiation for our considerations, and we shall be using it for various variables as well as t.)

Consider also the Virasoro algebra $\mathfrak{v}$, the well-known central extension

$$
0 \rightarrow \mathbb{C} c \rightarrow \mathfrak{v} \rightarrow \mathfrak{d} \rightarrow 0
$$

where $\mathfrak{v}$ has basis $\{L(n) \mid n \in \mathbb{Z}\}$ together with a central element $c$; the bracket relations among the $L(n)$ are given by

$$
[L(m), L(n)]=(m-n) L(m+n)+\frac{1}{12}\left(m^{3}-m\right) \delta_{m+n, 0} c
$$


and $L(n)$ maps to $-t^{n} D$ in 2.3). The number $\frac{1}{12}\left(m^{3}-m\right)$, being onehalf the binomial coefficient $\left(\begin{array}{c}m+1 \\ 3\end{array}\right)$, is closely related to a third derivative, which becomes visible when we use generating functions to write the bracket relations (2.4), as we review below. The Virasoro algebra is naturally $\mathbb{Z}$ graded, with $\operatorname{deg} L(n)=n$ and $\operatorname{deg} c=0$.

The following classical realization of the Lie algebra $\mathfrak{v}$ is well known: We start with the Heisenberg Lie algebra with basis consisting of the symbols $h(n)$ for $n \in \mathbb{Z}, n \neq 0$ and a central element 1 , with the bracket relations

$$
[h(m), h(n)]=m \delta_{m+n, 0} 1 .
$$

For convenience we adjoin an additional central basis element $h(0)$, so that the relations (2.5) hold for all $m, n \in \mathbb{Z}$. This Lie algebra acts irreducibly on the polynomial algebra

$$
S=\mathbb{C}[h(-1), h(-2), h(-3), \ldots]
$$

as follows: For $n<0, h(n)$ acts as the multiplication operator; for $n>0$, $h(n)$ acts as the operator $n \frac{\partial}{\partial h(-n)} ; h(0)$ acts as 0 ; and 1 acts as the identity operator. Then $\mathfrak{v}$ acts on $S$ by means of the following operators:

$$
\begin{gathered}
c \mapsto 1, \\
L(n) \mapsto \frac{1}{2} \sum_{j \in \mathbb{Z}} h(j) h(n-j) \text { for } n \neq 0, \\
L(0) \mapsto \frac{1}{2} \sum_{j \in \mathbb{Z}} h(-|j|) h(|j|) .
\end{gathered}
$$

It is important to observe that in the case of $L(0)$, the absolute values make the formal quadratic operator well defined, while for $n \neq 0$ the operator is well defined as it stands, since $[h(j), h(n-j)]=0$. Thus the operators (2.8) and (2.9) are in "normal-ordered form," that is, the "annihilation operators" $h(n)$ for $n>0$ act to the right of the "creation operators" $h(n)$ for $n<0$. Using colons to denote normal ordering (the appropriate reordering of the factors in the product if necessary), we thus have

$$
L(n) \mapsto \frac{1}{2}: \sum_{j \in \mathbb{Z}} h(j) h(n-j):
$$


for all $n \in \mathbb{Z}$.

It is an instructive and not-so-trivial (classical) exercise to verify by direct computation that the operators (2.10) indeed satisfy the bracket relations (2.4). (This exercise and the related constructions are presented in [FLM], for example, where the standard generalization of this construction of $\mathfrak{v}$ using a Heisenberg algebra based on a finite-dimensional space of operators $h(n)$ for each $n$ is also carried out.)

Vertex operator algebra theory and conformal field theory place this exercise into a very general, natural setting (among many other things), with conceptual approaches and techniques (cf. [FLM). It is standard procedure to embed operators such as $h(n)$ and $L(n)$ into generating functions and to compute with these generating functions, using a formal calculus, and to systematically avoid computing with the individual operators. Doing this vastly simplifies computations that would otherwise be complicated or sometimes almost impossible. In fact, we shall be using a number of generating-function ideas below.

The space $S$ carries a natural $\mathbb{Z}$-grading, determined by the rule deg $h(j)=$ $j$ for $j<0$. Then $S$ is in fact graded by the nonpositive integers, and the $\mathfrak{v}$-module $S$ is a graded module. It turns out to be appropriate to use the negative of this grading, that is, to define a new grading (by "conformal weights") on the space $S$ by the rule wt $h(-j)=j$ for $j>0$. One reason why this is natural is that for each $n \geq 0$, the homogeneous subspace of $S$ of weight $n$ coincides with the eigenspace of the operator $L(0)$ with eigenvalue $n$, as is easy to see. For $n \in \mathbb{Z}$ (or $n \geq 0$ ) we define $S_{n}$ to be the homogeneous subspace of $S$ of weight $n$, and we consider the formal power series in the formal variable $q$ given by

$$
\operatorname{dim}_{*} S=\sum_{n \geq 0}\left(\operatorname{dim} S_{n}\right) q^{n}
$$

(the "graded dimension" of the graded space $S$ ). Clearly, from the definitions,

$$
\operatorname{dim}_{*} S=\prod_{n>0}\left(1-q^{n}\right)^{-1}
$$

Here are the main points about these classical considerations that we want to emphasize: As is well known in conformal field theory, removing the normal ordering in the definition of the operator $L(0)$ introduces an infinity 
which formally equals $\frac{1}{2} \zeta(-1)$ : The unrigorous expression

$$
\bar{L}(0)=\frac{1}{2} \sum_{j \in \mathbb{Z}} h(-j) h(j)
$$

formally equals (by the bracket relations (2.5))

$$
L(0)+\frac{1}{2}(1+2+3+\cdots),
$$

which itself formally equals

$$
L(0)+\frac{1}{2} \zeta(-1)=L(0)-\frac{1}{24} .
$$

We rigorize $\bar{L}(0)$ by defining it as:

$$
\bar{L}(0)=L(0)+\frac{1}{2} \zeta(-1)
$$

and we define

$$
\bar{L}(n)=L(n) \text { for } n \neq 0,
$$

to get a new basis of the Lie algebra $\mathfrak{v}$. (We are identifying the elements of $\mathfrak{v}$ with operators on the space $S$.) The brackets become:

$$
[\bar{L}(m), \bar{L}(n)]=(m-n) \bar{L}(m+n)+\frac{1}{12} m^{3} \delta_{m+n, 0} ;
$$

that is, $m^{3}-m$ in (2.4) has become the pure monomial $m^{3}$.

Perhaps the most important (and also well-known) thing accomplished by this formal removal of the normal ordering is the following: We define a new grading of the space $S$ by using the eigenvalues of the modified operator $\bar{L}(0)$ in place of $L(0)$, so that the grading of $S$ is "shifted" from the previous grading by conformal weights by the subtraction of $\frac{1}{24}$ from the weights. We let $\chi(S)$ be the corresponding graded dimension, so that

$$
\chi(S)=\frac{1}{\eta(q)},
$$

where

$$
\eta(q)=q^{\frac{1}{24}} \prod_{n>0}\left(1-q^{n}\right) .
$$


The point is that $\eta(q)$ has important (classical) modular transformation properties, unlike $\prod_{n>0}\left(1-q^{n}\right)$, when viewed as a function of $\tau$ in the upper half-plane via the substitution $q=e^{2 \pi i \tau} ; \eta(q)$ is Dedekind's eta-function.

Bloch [BI] extended this classical story in various ways, in particular, the following: Instead of the Lie algebra $\mathfrak{d}$, we consider the larger Lie algbebra of formal differential operators, spanned by

$$
\left\{t^{n} D^{m} \mid n \in \mathbb{Z}, m \geq 0\right\}
$$

or more precisely, we restrict to $m>0$ and further, to the Lie subalgebra $\mathcal{D}^{+}$ spanned by the differential operators of the form $D^{r}\left(t^{n} D\right) D^{r}$ for $r \geq 0, n \in \mathbb{Z}$. Then we can construct a central extension of $\mathcal{D}^{+}$using generalizations of the normally-ordered quadratic operators (2.10) above:

$$
\begin{gathered}
L^{(r)}(n)=\frac{1}{2} \sum_{j \in \mathbb{Z}} j^{r} h(j)(n-j)^{r} h(n-j) \text { for } n \neq 0, \\
L^{(r)}(0)=\frac{1}{2} \sum_{j \in \mathbb{Z}}(-j)^{r} h(-|j|) j^{r} h(|j|),
\end{gathered}
$$

that is,

$$
L^{(r)}(n)=: \frac{1}{2} \sum_{j \in \mathbb{Z}} j^{r} h(j)(n-j)^{r} h(n-j):
$$

for $n \in \mathbb{Z}$. Direct computation of the commutators among these operators [BI] shows that they provide a central extension of $\mathcal{D}^{+}$such that

$$
L^{(r)}(n) \mapsto(-1)^{r+1} D^{r}\left(t^{n} D\right) D^{r}
$$

(cf. [KP]). (It is not surprising in retrospect that these operators $L^{(r)}(n)$ are related to differential operators, because the generating function of these operators as $n$ ranges through $\mathbb{Z}$ is based on $D^{r}$, as we discuss below.)

A central point of [B] is that the formal removal of the normal-ordering procedure in the definition (2.23) of $L^{(r)}(0)$ adds the infinity $(-1)^{r} \frac{1}{2} \zeta(-2 r-$ $1)=" \sum_{n>0} n^{2 r+1} "$ (generalizing (2.13)-(2.15)), and if we correspondingly define

$$
\bar{L}^{(r)}(0)=L^{(r)}(0)+(-1)^{r} \frac{1}{2} \zeta(-2 r-1)
$$

and $\bar{L}^{(r)}(n)=L^{(r)}(n)$ for $n \neq 0$ (generalizing (2.16) and (2.17)), the commutators simplify in a remarkable way: As direct computation [B] shows, the 
complicated polynomial in the scalar term of $\left[\bar{L}^{(r)}(m), \bar{L}^{(s)}(-m)\right]$ reduces to a pure monomial in $m$, by analogy with, and generalizing, the passage from $m^{3}-m$ to $m^{3}$ in (2.18). The precise formulas can be found in $[\mathrm{B}]$, along with further results; for instance, in [B] , these considerations and results are generalized to Dirichlet $L$-series in place of the zeta function.

\section{First "explanation" and generalization}

Our goal is to present two layers of "explanation" and generalization of the results of [B]] sketched above. First we need some elementary formal background:

What does $\zeta(-2 r-1)$ "mean," for a nonnegative integer $r$ ?

It is a well-known classical fact that for $k>1$,

$$
\zeta(-k+1)=-\frac{B_{k}}{k}
$$

where the $B_{k}$ are the Bernoulli numbers, defined by the generating function

$$
\frac{x}{e^{x}-1}=\sum_{k \geq 0} \frac{B_{k}}{k !} x^{k},
$$

where $x$ is a formal variable. This formal power series in $x$ is understood to be computed (on the left-hand side) by expanding $e^{x}-1$ as the formal series $x+\frac{x^{2}}{2 !}+\cdots$ and performing the division of formal power series to obtain a formal power series with constant term 1; this of course corresponds to expanding a complex function in a certain domain, but we are operating purely formally.

Why does $B_{k}$ defined in this way have anything to do with the formal series $\sum_{n>0} n^{k-1}$ ? We recall Euler's heuristic interpretation of such formal sums as " $1+2+\cdots$ " (cf. the Preface of [Hi]); actually, we give a variant of Euler's interpretation adapted to the main theme that we shall introduce:

Consider the expansion (from (3.2))

$$
\frac{1}{1-e^{x}}=-\sum_{k \geq 0} \frac{B_{k}}{k !} x^{k-1} .
$$


Expand the left-hand side unrigorously as the formal geometric series

$$
1+e^{x}+e^{2 x}+\cdots=1+\sum_{k \geq 0} \frac{1^{k}}{k !} x^{k}+\sum_{k \geq 0} \frac{2^{k}}{k !} x^{k}+\cdots
$$

For $k>1$, the coefficient of $x^{k-1}$ in this formal expression is

$$
\frac{1}{(k-1) !}\left(1^{k-1}+2^{k-1}+\cdots\right)
$$

which looks like $\frac{1}{(k-1) !} \zeta(-k+1)$. Also, the coefficient of $x^{0}$ in (3.4) is formally $1+\frac{1}{0 !}\left(1^{0}+2^{0}+\cdots\right)$, which we formally view as $1+\zeta(0)$ (and not as $\zeta(0)$ ). Thus, formally equating the coefficients of $x^{l}$ for $l \geq 0$ in (3.2) "explains" (3.1) and the fact that $\zeta(0)=-B_{1}-1\left(=-\frac{1}{2}\right)$; now we know what (1.4) says.

The key point here is the interplay between the formal geometric series expansion (in powers of $e^{x}$ ) and the expansion in powers of $x$.

Now, how do we interpret all of this via vertex operator algebra theory?

First note that the expressions $(2.22)-(2.24)$ above for $L^{(r)}(n)$ suggest $r^{\text {th }}$ derivatives. We have already mentioned that a basic theme in vertex operator algebra theory is to always use appropriate generating functions (as we just did, incidentally, in the heuristic discussion above). First we put our individual operators into generating functions. Using a formal variable $x$, we define

$$
h(x)=\sum_{n \in \mathbb{Z}} h(n) x^{-n}
$$

and

$$
L^{(r)}(x)=\sum_{n \in \mathbb{Z}} L^{(r)}(n) x^{-n},
$$

and using $D_{x}$ to denote the operator $x \frac{d}{d x}$ (recall the comment after (2.2)), we observe that

$$
L^{(r)}(x)=\frac{1}{2}:\left(D_{x}^{r} h(x)\right)^{2}:
$$

where the colons, as always, denote normal ordering (recall (2.10)). (For other purposes, other versions of these generating functions are used, in particular, $h(x)=\sum_{n \in \mathbb{Z}} h(n) x^{-n-1}$, as in (4.14) below, in place of (3.6), but we have chosen the appropriate generating functions for our purposes.) 
Using standard elementary techniques, we could directly compute the brackets $\left[L^{(r)}\left(x_{1}\right), L^{(s)}\left(x_{2}\right)\right]$ of these generating functions, for $r, s \geq 0$, where $x_{1}$ and $x_{2}$ are independent commuting formal variables. (As always in vertex operator algebra theory or conformal field theory, when we consider such operations as brackets of generating functions, we need independent commuting formal variables; the expression $\left[L^{(r)}(x), L^{(s)}(x)\right]$, with the variable $x$ repeated, would be meaningless.) But this computation, which might be carried out as a more complicated variant of the argument on pp. 224-226 of [FLM], for example, would not be simple. It would of course recover the information of the brackets $\left[L^{(r)}(m), L^{(s)}(n)\right]$ computed in $\left.\mathbb{B}\right]$.

The best use of generating functions in this context is instead to also introduce suitable generating functions over the number of derivatives. Consider the elementary formal Taylor theorem equating the application of a formal exponential of a formal multiple of $\frac{d}{d x}$ with a formal substitution operation:

$$
e^{y \frac{d}{d x}} f(x)=f(x+y)
$$

where $f(x)$ is an arbitrary formal series of the form $\sum_{n} a_{n} x^{n}$, and where it is understood that each binomial expression $(x+y)^{n}$ is to be expanded in nonnegative integral powers of $y$. Here $n$ is allowed to range over something very general, like $\mathbb{Z}$ or even $\mathbb{C}$, say, and the $a_{n}$ lie in a fixed vector space; $f(x)$ is very definitely not necessarily the expansion of an analytic function. Formula (3.9) is proved by direct formal expansion of both sides (cf. [FLM, Proposition 8.3.1; Taylor's theorem in this kind of generality is heavily exploited in Chapter 8 of [FLM, for instance). Now $\frac{d}{d x}$ is of course a formal infinitesimal translation (as (3.9) states), but for our present purposes we want the following formal multiplicative analogue of (3.9):

$$
e^{y D_{x}} f(x)=f\left(e^{y} x\right),
$$

with $f(x)$ as above (again cf. [FLM], Proposition 8.3.1), which expresses the fact that $D_{x}$ is a formal infinitesimal dilation.

Now : $\left(D_{x}^{r} h(x)\right)^{2}$ : (recall (3.8)) is hard to put into a "good" generating function over $r$, but we can make the problem easier by making it more general: Consider independently many derivatives on each of the two factors $h(x)$ in $: h(x)^{2}$ :, use two new independent formal variables $y_{1}$ and $y_{2}$, and form the generating function

$$
L^{\left(y_{1}, y_{2}\right)}(x)=\frac{1}{2}:\left(e^{y_{1} D_{x}} h(x)\right)\left(e^{y_{2} D_{x}} h(x)\right):=\frac{1}{2}: h\left(e^{y_{1}} x\right) h\left(e^{y_{2}} x\right):
$$


(where we use (3.10)), so that $L^{(r)}(x)$ is a "diagonal piece" of this generating function in the sense that it is $(r !)^{2}$ times the coefficient of $y_{1}^{r} y_{2}^{r}$ in $L^{\left(y_{1}, y_{2}\right)}(x)$. Using formal vertex operator calculus techniques (generalizing the argument on pp. 224-226 of [FLM], for example), we can calculate

$$
\left[: h\left(e^{y_{1}} x_{1}\right) h\left(e^{y_{2}} x_{1}\right):,: h\left(e^{y_{3}} x_{2}\right) h\left(e^{y_{4}} x_{2}\right):\right] .
$$

Then, a nontrivial, and in fact quite tricky, vertex operator computation recovers Bloch's formulas, in somewhat generalized form, as we explain next. Here are the main points:

The expression $h(x)^{2}$ is not rigorous (as we observe for instance by trying to compute the coefficient of any fixed power of $x$ in this expression), while the normal-ordered expression $: h(x)^{2}$ : is of course rigorous. The expression $h\left(e^{y_{1}} x\right) h\left(e^{y_{2}} x\right)$ is still not rigorous (even though the expressions $e^{y_{1}} x$ and $e^{y_{2}} x$ are distinct), as we see by (for example) trying to compute the constant term in the variables $y_{1}$ and $y_{2}$ in this expression. The lack of rigor in this expression in fact corresponds exactly to the occurrence of formal sums like $\sum_{n>0} n^{r}$ with $r>0$, as we have been discussing above.

However, we have

$$
h\left(x_{1}\right) h\left(x_{2}\right)=: h\left(x_{1}\right) h\left(x_{2}\right):+x_{2} \frac{\partial}{\partial x_{2}} \frac{1}{1-x_{2} / x_{1}}
$$

(an exercise using elementary vertex operator techniques), and it follows that

$$
h\left(e^{y_{1}} x_{1}\right) h\left(e^{y_{2}} x_{2}\right)=: h\left(e^{y_{1}} x_{1}\right) h\left(e^{y_{2}} x_{2}\right):+x_{2} \frac{\partial}{\partial x_{2}} \frac{1}{1-e^{y_{2}} x_{2} / e^{y_{1}} x_{1}} .
$$

Note that $x_{2} \frac{\partial}{\partial x_{2}}$ can be replaced by $-\frac{\partial}{\partial y_{1}}$ in the last expression (and this is one illustration of the naturalness of our emphasis on the "homogeneous" differential operator $D_{x}=x \frac{\partial}{\partial x}$ rather than $\left.\frac{\partial}{\partial x}\right)$. The expression $\frac{1}{1-e^{y_{2}} x_{2} / e^{y_{1}} x_{1}}$ came from, and is, a geometric series expansion (recall (3.13)).

Now we try to set $x_{1}=x_{2}(=x)$ in $(3.14)$. The result of this procedure is unrigorous on the left-hand side, as we have pointed out above, but the result has rigorous meaning on the right-hand side, because the normalordered product $: h\left(e^{y_{1}} x\right) h\left(e^{y_{2}} x\right)$ : is certainly well defined, and the expression $-\frac{\partial}{\partial y_{1}} \frac{1}{1-e^{-y_{1}+y_{2}}}$ can be interpreted rigorously as in (3.2) and (3.3); more precisely (the role of $x$ in (3.3) being played here by $-y_{1}+y_{2}$ ), we take $\frac{1}{1-e^{-y_{1}+y_{2}}}$ 
to mean the formal (Laurent) series in $y_{1}$ and $y_{2}$ of the shape

$$
\frac{1}{1-e^{-y_{1}+y_{2}}}=\left(y_{1}-y_{2}\right)^{-1} F\left(y_{1}, y_{2}\right)
$$

where $\left(y_{1}-y_{2}\right)^{-1}$ is understood as the binomial expansion (geometric series) in nonnegative powers of $y_{2}$ and $F\left(y_{1}, y_{2}\right)$ is an (obvious) formal power series in (nonnegative powers of) $y_{1}$ and $y_{2}$. This motivates us to define a new "normal-ordering" procedure

$$
{ }_{+}^{+} h\left(e^{y_{1}} x\right) h\left(e^{y_{2}} x\right)_{+}^{+}=: h\left(e^{y_{1}} x\right) h\left(e^{y_{2}} x\right):-\frac{\partial}{\partial y_{1}} \frac{1}{1-e^{-y_{1}+y_{2}}},
$$

with the last part of the right-hand side being understood as we just indicated. Again compare this with the heuristic discussion above; this expression came from a geometric series, but it becomes rigorous only when we expand in the new way (actually, we might alternatively replace the binomial expansion $\left(y_{1}-y_{2}\right)^{-1}$ by the different expansion of the same formal expression in nonnegative powers of $y_{1}$ rather than of $y_{2}$, but it is more natural to make the choice that we did).

Formula (3.16) and its indicated interpretation give a natural "explanation" of the zeta-function-modified operators defined in (2.26): We use (3.16) to define the following analogues of the operators (3.11):

$$
\bar{L}^{\left(y_{1}, y_{2}\right)}(x)=\frac{1}{2}{ }_{+}^{+} h\left(e^{y_{1}} x\right) h\left(e^{y_{2}} x\right)_{+}^{+},
$$

and it is easy to check that the operator $\bar{L}^{(r)}(n)$ is exactly $(r !)^{2}$ times the coefficient of $y_{1}^{r} y_{2}^{r} x_{0}^{-n}$ in (3.17); the significant case is the case $n=0$.

We are now ready to formulate the result mentioned just after (3.12) above. With the new normal ordering (3.16) replacing the old one, remarkable cancellation occurs in the commutator (3.12), and the result is:

Theorem 3.1 With the formal delta-function Laurent series $\delta(x)$ defined as

$$
\delta(x)=\sum_{n \in \mathbb{Z}} x^{n}
$$

and with independent commuting formal variables as indicated, we have:

$$
\left[\bar{L}^{\left(y_{1}, y_{2}\right)}\left(x_{1}\right), \bar{L}^{\left(y_{3}, y_{4}\right)}\left(x_{2}\right)\right]
$$




$$
\begin{aligned}
=- & \frac{1}{2} \frac{\partial}{\partial y_{1}}\left(\bar{L}^{\left(-y_{1}+y_{2}+y_{3}, y_{4}\right)}\left(x_{2}\right) \delta\left(\frac{e^{y_{1}} x_{1}}{e^{y_{3}} x_{2}}\right)\right. \\
& \left.+\bar{L}^{\left(-y_{1}+y_{2}+y_{4}, y_{3}\right)}\left(x_{2}\right) \delta\left(\frac{e^{y_{1}} x_{1}}{e^{y_{4}} x_{2}}\right)\right) \\
& -\frac{1}{2} \frac{\partial}{\partial y_{2}}\left(\bar{L}^{\left(y_{1}-y_{2}+y_{3}, y_{4}\right)}\left(x_{2}\right) \delta\left(\frac{e^{y_{2}} x_{1}}{e^{y_{3}} x_{2}}\right)\right. \\
& \left.+\bar{L}^{\left(y_{1}-y_{2}+y_{4}, y_{3}\right)}\left(x_{2}\right) \delta\left(\frac{e^{y_{2}} x_{1}}{e^{y_{4}} x_{2}}\right)\right) .
\end{aligned}
$$

Actually, hidden in the right-hand side of (3.19) are formal expressions (suitably expanded) of the type $\left(y_{1}-y_{2}-y_{3}+y_{4}\right)^{-3}$ because of the formal pole $y_{1}=y_{2}$ in (3.16), and these expressions, multiplied by the formal deltafunction expressions, are the source of the pure monomials in $m$ that we set out to explain (recall the end of Section 2). Indeed, the delta-function expression $\delta\left(e^{y_{1}} x_{1} / e^{y_{3}} x_{2}\right)$, for instance, can be written as $e^{y_{1} D_{x_{1}}} e^{y_{3} D_{x_{2}}} \delta\left(x_{1} / x_{2}\right)$, and when we extract and equate the coefficients of the monomials in the variables $y_{1}^{r} y_{2}^{r} y_{3}^{s} y_{4}^{s}$ on the two sides of (3.19), we get expressions like $\left(D^{j} \delta\right)\left(x_{1} / x_{2}\right)$, whose expansion, in turn, in powers of $x_{1}$ and $x_{2}$ clearly yields a pure monomial analogous to and generalizing the expression $m^{3}$ in (2.18). In this way, we have an explicit generalization and explanation of Bloch's formula for $\left[\bar{L}^{(r)}(m), \bar{L}^{(s)}(n)\right]$ in terms of the operators $\bar{L}^{(j)}(m+n)$ and a monomial in $m$.

We have been working all along with a Heisenberg algebra based on a one-dimensional space - that is, a Heisenberg algebra with only one dimension of operators, spanned by the element $h(n)$, for each $n$, but all of these considerations hold equally well in the more general situation where we start with a Heisenberg algebra based on a finite-dimensional space.

All of this has been an interesting (and nontrivial) special case of vertex operator algebra theory, but what is really happening?

\section{Second "explanation" and generalization}

It turns out that Theorem 3.1 is an extremely special case of a something that can be formulated and proved for an arbitrary vertex operator algebra (and indeed this gives another motivation for the general theory). We recall the definition of the notion of vertex (operator) algebra from [B], [FLM and 
[FHI]; the principles that we have found are based heavily on the "Jacobi identity" as formulated in [FLM and [FHD:

Definition 4.1 A vertex operator algebra $(V, Y, \mathbf{1}, \omega)$, or simply $V$ (over $\mathbb{C}$ ), is a $\mathbb{Z}$-graded vector space (graded by weights)

$$
V=\coprod_{n \in \mathbb{Z}} V_{(n)} ; \text { for } v \in V_{(n)}, n=\mathrm{wt} v ;
$$

such that

$$
\begin{gathered}
\operatorname{dim} V_{(n)}<\infty \text { for } n \in \mathbb{Z}, \\
V_{(n)}=0 \text { for } n \text { sufficiently small, }
\end{gathered}
$$

equipped with a linear map $V \otimes V \rightarrow V\left[\left[x, x^{-1}\right]\right]$, or equivalently,

$$
\begin{aligned}
V & \rightarrow(\text { End } V)\left[\left[x, x^{-1}\right]\right] \\
v & \mapsto Y(v, x)=\sum_{n \in \mathbb{Z}} v_{n} x^{-n-1} \quad\left(\text { where } v_{n} \in \text { End } V\right)
\end{aligned}
$$

$Y(v, x)$ denoting the vertex operator associated with $v$, and equipped also with two distinguished homogeneous vectors $\mathbf{1} \in V_{(0)}$ (the vacuum) and $\omega \in V_{(2)}$. The following conditions are assumed for $u, v \in V$ : the lower truncation condition holds:

$$
u_{n} v=0 \text { for } n \text { sufficiently large }
$$

(or equivalently, $Y(u, x) v$ involves only finitely many negative powers of $x$ );

$$
Y(\mathbf{1}, x)=1 \text { (1 on the right being the identity operator); }
$$

the creation property holds:

$$
Y(v, x) \mathbf{1} \in V[[x]] \text { and } \lim _{x \rightarrow 0} Y(v, x) \mathbf{1}=v
$$

(that is, $Y(v, x) \mathbf{1}$ involves only nonnegative integral powers of $x$ and the constant term is $v$ ); with $\delta(x)$ as in (3.18) and with binomial expressions understood (as above) to be expanded in nonnegative powers of the second variable, the Jacobi identity (the main axiom) holds:

$$
\begin{gathered}
x_{0}^{-1} \delta\left(\frac{x_{1}-x_{2}}{x_{0}}\right) Y\left(u, x_{1}\right) Y\left(v, x_{2}\right)-x_{0}^{-1} \delta\left(\frac{x_{2}-x_{1}}{-x_{0}}\right) Y\left(v, x_{2}\right) Y\left(u, x_{1}\right) \\
=x_{2}^{-1} \delta\left(\frac{x_{1}-x_{0}}{x_{2}}\right) Y\left(Y\left(u, x_{0}\right) v, x_{2}\right)
\end{gathered}
$$


(note that when each expression in (4.8) is applied to any element of $V$, the coefficient of each monomial in the formal variables is a finite sum; on the right-hand side, the notation $Y\left(\cdot, x_{2}\right)$ is understood to be extended in the obvious way to $\left.V\left[\left[x_{0}, x_{0}^{-1}\right]\right]\right)$; the Virasoro algebra relations hold (acting on $V)$ :

$$
[L(m), L(n)]=(m-n) L(m+n)+\frac{1}{12}\left(m^{3}-m\right) \delta_{n+m, 0}(\operatorname{rank} V) 1
$$

for $m, n \in \mathbb{Z}$, where

$$
L(n)=\omega_{n+1} \text { for } n \in \mathbb{Z}, \text { i.e., } Y(\omega, x)=\sum_{n \in \mathbb{Z}} L(n) x^{-n-2}
$$

and

$$
\begin{gathered}
\operatorname{rank} V \in \mathbb{C} \\
L(0) v=n v=(\text { wt } v) v \text { for } n \in \mathbb{Z} \text { and } v \in V_{(n)} \\
\frac{d}{d x} Y(v, x)=Y(L(-1) v, x)
\end{gathered}
$$

(the $L(-1)$-derivative property).

Note that as in Theorem 3.1, the Jacobi identity encapsulates higher derivatives of delta-function expressions, since the expression $\delta\left(\left(x_{1}-x_{2}\right) / x_{0}\right)$, say, can be expanded by means of (3.9). The use of the three formal variables, rather than complex variables (which would require changes in the formulas), allows the full symmetry of the Jacobi identity to reveal itself (see [FLM and [FHD).

The polynomial algebra $S$ (recall (2.6)) carries a canonical vertex operator algebra structure of rank 1 with vacuum vector 1 equal to $1 \in S$ and with the operators $L(n)$ agreeing with the operators defined in Section 2 (cf. [FLM]). We will not describe the vertex operators $Y(v, x)$ here, except to say that

$$
Y(h(-1), x)=x^{-1} h(x)=\sum_{n \in \mathbb{Z}} h(n) x^{-n-1}
$$

(recall (3.6)) and that the element $\omega$ is $\frac{1}{2}(h(-1))^{2} \in S$.

If we omit the grading and the Virasoro algebra from Definition 4.1 and adjust the axioms appropriately, we have the notion of "vertex algebra" as 
introduced in $[\mathrm{Bg}]$, except that Borcherds used certain special cases of the Jacobi identity instead of (4.8). The identity (4.8) is the canonical "maximal" axiom: It contains the "full" necessary information in compact form; it is analogous to the classical Jacobi identity in the definition of the notion of Lie algebra; and it is invariant in a natural sense under the symmetric group on three letters (see [FLM and [FHL]).

There are also "minimal" axioms, stemming from the fact that the (suitably formulated) "commutativity" of the operators $Y\left(u, x_{1}\right)$ and $Y\left(v, x_{2}\right)$ implies "associativity" (again suitably formulated) and hence the Jacobi identity (see [FLM] and [FHप]; cf. [BPZ and $[\mathrm{G}]$ ). The simplest "minimal" axiom, as found in [DD (actually, in the greater generality of "abelian intertwining algebras") states that for $u, v \in V$, there exists $n \geq 0$ such that

$$
\left(x_{1}-x_{2}\right)^{n}\left[Y\left(u, x_{1}\right), Y\left(v, x_{2}\right)\right]=0
$$

(see [DD], formula (1.4)). However, it is still a nontrivial matter to construct examples, even relatively simple ones like $S$, of vertex operator algebras, partly because one has to extend the condition (4.15) from generators to arbitrary elements of $V$. A general and systematic approach and solution to this and related problems was first found by Li (see [Li1], [Li2]).

The commutativity condition asserts that for $u, v \in V$,

$$
Y\left(u, x_{1}\right) Y\left(v, x_{2}\right) \sim Y\left(v, x_{2}\right) Y\left(u, x_{1}\right),
$$

where " " denotes equality up to a suitable kind of generalized analytic continuation, and the associativity condition asserts that

$$
Y\left(u, x_{1}\right) Y\left(v, x_{2}\right) \sim Y\left(Y\left(u, x_{1}-x_{2}\right) v, x_{2}\right),
$$

where the right-hand side and the generalized analytic continuation have to be understood in suitable ways (again see [FLM and [FHL and cf. BPZ and $[\mathrm{G} \|)$; the right-hand side of (4.17) is not a well-defined formal series in $x_{1}$ and $x_{2}$.

On the level of these basic principles, for any vertex operator algebra $V$ we shall now conceptually formulate and considerably generalize the normalordering procedure (3.16) and we shall formulate a new general "Jacobi identity" which implies Theorem 3.1 in the very particular case of the vertex operator algebra $S$ and very special vertex operators. Ideas in Zhu's work [Z1], [Z2] enter into our considerations. 
Formally replacing $x_{1}$ by $e^{y} x_{2}$ in (4.17), we find (formally and unrigorously) that

$$
Y\left(u, e^{y} x_{2}\right) Y\left(v, x_{2}\right) \sim Y\left(Y\left(u,\left(e^{y}-1\right) x_{2}\right) v, x_{2}\right) .
$$

Now we observe that while the left-hand side of (4.18) is not a well-defined formal series in the formal variables $y$ and $x_{2}$, the right-hand side of (4.18) is in fact a well-defined formal series in these formal variables. By replacing $x_{1}$ by $e^{y} x_{2}$ we have made the right-hand side of (4.18) rigorous (and the left-hand side unrigorous). This situation should be compared with our motivation for introducing the normal-ordering procedure (3.16) above.

Next, instead of the vertex operators $Y(v, x)$, we want the modified vertex operators defined for homogeneous elements $v \in V$ by:

$$
X(v, x)=x^{\mathrm{wt} v} Y(v, x)=Y\left(x^{L(0)} v, x\right),
$$

as in FLM, formula (8.5.27) (recall that $L(0)$-eigenvalues define the grading of $V$ ); the formula $X(v, x)=Y\left(x^{L(0)} v, x\right)$ works for all $v \in V$ (not necessarily homogeneous). For instance, the operator $h(x)$ (3.6) is exactly $X(h(-1), x)$ (cf. (4.14)).

What we really want is a rigorous expression that "equals" the unrigorous expression $X\left(u, e^{y} x_{2}\right) X\left(v, x_{2}\right)$; this will considerably generalize our interpretation of the unrigorous expression $h\left(e^{y} x_{2}\right) h\left(x_{2}\right)$ (cf. (3.14) and (3.16)). So we replace $u$ by $\left(e^{y} x_{2}\right)^{L(0)} u$ and $v$ by $x_{2}^{L(0)} v$ in (4.18) and using basic techniques we get:

$$
X\left(u, e^{y} x_{2}\right) X\left(v, x_{2}\right) \sim X\left(Y\left(e^{y L(0)} u, e^{y}-1\right) v, x_{2}\right),
$$

and this right-hand side is still rigorous (and the left-hand side still unrigorous). But $Y\left(e^{y L(0)} u, e^{y}-1\right)$ is exactly Zhu's operator $Y[u, y]$ in [Z1], [Z2], so that

$$
X\left(u, e^{y} x_{2}\right) X\left(v, x_{2}\right) \sim X\left(Y[u, y] v, x_{2}\right) .
$$

By Zhu's change-of-variables theorem, $x \mapsto Y[u, x]$ defines a new vertex operator algebra structure on the same vector space $V$ under suitable conditions; this theorem was a step in Zhu's vertex-operator-algebraic proof of the modular-invariance properties of "characters" (cf. the comments surrounding (2.19) above). There have been two subsequent treatments of this change-of-variables theorem, in [L1] and in [H1], [H2]; in the latter works, 
Huang considerably generalized this result (and removed a hypothesis of Zhu's) using his geometric analysis of the Virasoro algebra structure in a vertex operator algebra. The formal relation (4.21) generalizes to products of several operators.

So we want to bracket the (rigorous) expressions $X(Y[u, y] v, x)$, which are the correct natural generalization of the expression (3.16) above (at least with $y_{2}=1$ in (3.16), but this restriction is a minor issue since $y_{2}$ can easily be restored). Keep in mind that the formal relation (4.21) naturally implements the formal relation (1.4) in a foundational way from the viewpoint of vertex operator algebras.

But just as in [FLM], where the Jacobi identity for vertex operator algebras was the most natural general formula, here we find that the most natural thing to do is to formulate and prove a new Jacobi identity, based on the operators $X(Y[u, y] v, x)$, in the general setting of an arbitrary vertex (operator) algebra, rather than just to bracket the operators. It turns out that delta-function expressions of the type appearing on the right-hand side of (3.19), and that in turn "explained" the phenomenon of pure monomials in $m$ (as discussed above), arise naturally in complete generality, and when we ask for a Jacobi identity rather than just a commutator formula in general, we find that delta-function expressions of this same type appear on the left-hand side as well as the right-hand side. This is another instance of the naturalness of "Jacobi identities," which have symmetries that commutator formulas do not have. We state our result for the operators $X(v, x)$ rather than $X(Y[u, y] v, x)$ (i.e., the case where $u$ is the vacuum vector):

Theorem 4.2 In any vertex (operator) algebra $V$, for $u, v \in V$ we have:

$$
\begin{gathered}
x_{0}^{-1} \delta\left(e^{y_{21}} \frac{x_{1}}{x_{0}}\right) X\left(u, x_{1}\right) X\left(v, x_{2}\right)-x_{0}^{-1} \delta\left(-e^{y_{12}} \frac{x_{2}}{x_{0}}\right) X\left(v, x_{2}\right) X\left(u, x_{1}\right) \\
=x_{2}^{-1} \delta\left(e^{y_{01}} \frac{x_{1}}{x_{2}}\right) X\left(Y\left[u,-y_{01}\right] v, x_{2}\right)
\end{gathered}
$$

where

$$
y_{21}=\log \left(1-\frac{x_{2}}{x_{1}}\right), \quad y_{12}=\log \left(1-\frac{x_{1}}{x_{2}}\right), \quad y_{01}=\log \left(1-\frac{x_{0}}{x_{1}}\right) .
$$

If we want the commutator $\left[X\left(u, x_{1}\right), X\left(v, x_{2}\right)\right]$, we simply extract the coefficient of $x_{0}^{-1}$ (the formal residue in the variable $x_{0}$ ) on both sides, and it 
turns out that the resulting right-hand side can be put into an elegant form. If we replace $u$ and $v$ by expressions of the shape $Y[u, y] v$, we obtain naturally a formula that generalizes formula (3.19) (Theorem 3.1) to arbitrary elements of arbitrary vertex (operator) algebras. That is, interesting as they are, the phenomena that we have been discussing concerning central extensions of Lie algebras of differential operators form extremely special cases of general vertex-operator-algebraic phenomena. The detailed formulations and proofs, and generalizations, are found in [L2]. Also, my student Antun Milas has generalized some of these results in a number of directions.

\section{References}

[BPZ] A. A. Belavin, A. M. Polyakov and A. B. Zamolodchikov, Infinite conformal symmetries in two-dimensional quantum field theory, Nucl. Phys. B241 (1984), 333-380.

[Bl] S. Bloch, Zeta values and differential operators on the circle, $J$. Algebra 182 (1996), 476-500.

[Bo] R. E. Borcherds, Vertex algebras, Kac-Moody algebras, and the Monster, Proc. Natl. Acad. Sci. USA 83 (1986), 3068-3071.

[DKM] E. Date, M. Kashiwara and T. Miwa, Vertex operators and $\tau$ functions - transformation groups for soliton equations II, Proc. Japan Acad. Ser. A Math. Sci. 57 (1981), 387-392.

[DL] C. Dong and J. Lepowsky, Generalized Vertex Algebras and Relative Vertex Operators, Progress in Math., Vol. 112, Birkhäuser, Boston, 1993.

[FKRW] E. Frenkel, V. Kac, A. Radul and W. Wang, $W_{1+\infty}$ and $W\left(g l_{N}\right)$ with central charge N, Comm. Math. Physics 170 (1995), 337-357.

[FHL] I. B. Frenkel, Y.-Z. Huang and J. Lepowsky, On axiomatic approaches to vertex operator algebras and modules, preprint, 1989; Memoirs Amer. Math. Soc. 104, 1993. 
[FLM] I. B. Frenkel, J. Lepowsky and A. Meurman, Vertex Operator Algebras and the Monster, Pure and Appl. Math., Vol. 134, Academic Press, Boston, 1988.

[G] P. Goddard, Meromorphic conformal field theory, Infinite Dimensional Lie Algebras and Groups, Advanced Series in Math. Physics, Vol. 7, ed. V. Kac, World Scientific, Singapore, 1989, 556-587.

[Hi] H. Hida, Elementary Theory of L-functions and Eisenstein Series, London Math. Soc. Student Texts, Vol. 26, Cambridge University Press, Cambridge, 1993.

[H1] Y.-Z. Huang, Applications of the geometric interpretation of vertex operator algebras, Proc. 20th International Conference on Differential Geometric Methods in Theoretical Physics, New York, 1991, ed. S. Catto and A. Rocha, World Scientific, Singapore, 1992, 333-343.

[H2] Y.-Z. Huang, Two-dimensional Conformal Geometry and Vertex Operator Algebras, Progress in Math., Vol. 148, Birkhäuser, Boston, 1997.

[KP] V. Kac and D. Peterson, Spin and wedge representations of infinitedimensional Lie algebras and groups, Proc. Natl. Acad. Sci. USA 78 (1981), 3308-3312.

[KR] V. Kac and A. Radul, Quasifinite highest weight modules over the Lie algebra of differential operators on the circle, Comm. Math. Physics 157 (1993), 429-457.

[L1] J. Lepowsky, Remarks on vertex operator algebras and moonshine, Proc. 20th International Conference on Differential Geometric Methods in Theoretical Physics, New York, 1991, ed. S. Catto and A. Rocha, World Scientific, Singapore, 1992, 362-370.

[L2] J. Lepowsky, A "Jacobi identity" for vertex operator algebras related to zeta-function values, to appear.

[Li1] H. Li, Local systems of vertex operators, vertex superalgebras and modules, preprint, 1993; J. Pure Appl. Alg. 109 (1996), 143-195. 
[Li2] H. Li, Local systems of twisted vertex operators, vertex superalgebras and twisted modules, Contemporary Math. 193 (1996), 203236 .

[M] Y. Matsuo, Free fields and quasi-finite representations of $W_{1+\infty}$, Physics Lett. B 326 (1994), 95-100.

[Z1] Y. Zhu, Vertex operators, elliptic functions and modular forms, Ph.D. thesis, Yale University, 1990.

[Z2] Y. Zhu, Modular invariance of characters of vertex operator algebras, J. Amer. Math. Soc. 9 (1996), 237-307.

Department of Mathematics, Rutgers University, Piscataway, NJ 08854

E-mail address: lepowsky@math.rutgers.edu 